\title{
Chandra and RXTE spectroscopy of the accreting msec pulsar IGR J00291+5934
}

\author{
A. Paizis ${ }^{1,2}$, M. A. Nowak ${ }^{3}$, J. Wilms ${ }^{4}$, T. J-L. Courvoisier ${ }^{1,5}$, K. Ebisawa ${ }^{6}$, J. Rodriguez $^{7,1}$, and P. Ubertini ${ }^{8}$ \\ 1 INTEGRAL Science Data Centre, Chemin d'Ecogia 16, 1290 Versoix, Switzerland \\ e-mail: Ada.Paizis@obs.unige.ch \\ 2 INAF-IASF, Sezione di Milano, Via Bassini 15, 20133 Milano, Italy \\ 3 Center for Space Research, MIT, Cambridge, MA, USA \\ 4 Department of Physics, University of Warwick, Coventry, CV4 7AL, UK \\ 5 Observatoire de Genève, 51 chemin des Mailletes, 1290 Sauverny, Switzerland \\ 6 NASA Goddard Space Flight Center, Code 662, Greenbelt, MD 20771, USA \\ 7 CEA Saclay, DSM/DAPNIA/SAp (CNRS UMR 7158 AIM), 91191 Gif-Sur-Yvette, France \\ 8 INAF-IASF, Sezione di Roma, via del Fosso del Cavaliere 100, 00133 Roma, Italy
}

Received 12 May 2005 / Accepted 14 July 2005

\section{ABSTRACT}

We report on an observation of the recently discovered accreting millisecond X-ray pulsar IGR J00291+5934 performed with the RXTEProportional Counter Array (PCA) and Chandra-High Energy Transmission Grating Spectrometer (HETGS). The RXTE data are from a twoweek follow-up of the source, while the Chandra observation took place around the end of the follow-up, about 12 days after the discovery of the source, when the source flux had decreased already by a factor of ten. The analysis of the Chandra data allowed us to extract the most precise X-ray position of IGR J00291 $+5934, \mathrm{RA}=00^{\mathrm{h}} 29^{\mathrm{m}} 03.08^{\mathrm{s}}$, and Dec $=+59^{\circ} 34^{\prime} 19.2^{\prime \prime}\left(0.6^{\prime \prime}\right.$ error $)$, compatible with the optical and radio ones. We find that the spectra of IGR J00291+5934 can be described by a combination of a thermal component and a power-law. Along the outburst detected by PCA, the power-law photon index showed no particular trend, while the thermal component $(\sim 1 \mathrm{keV}$, interpreted as a hot spot on the neutron star surface) became weaker until non-detection. In the simultaneous observation of the weak Chandra /RXTE spectrum, there was no longer any indication of the $\sim 1 \mathrm{keV}$ thermal component, while we detected a colder thermal component $(\sim 0.4 \mathrm{keV})$ that we interpret as the emission from the cold disc. A hint of a $6.4 \mathrm{keV}$ iron line was detected, together with an excess around $6.8 \mathrm{keV}$ and absorption feature around $7.1 \mathrm{keV}$. The last two features have never been detected in the spectra of accretion-driven millisecond pulsars before and, if confirmed, would suggest the presence of an expanding hot corona with high outflow velocities.

Key words. pulsars: individual: IGR J00291+5934

\section{Introduction}

In Low Mass X-Ray Binaries (LMXRB) hosting a neutron star, the accreting star is thought to be very old and weakly magnetised, compared to neutron star High Mass X-Ray Binaries. It is believed that in LMXRBs, the accretion disc is generally not influenced by the magnetic field and can extend very close to the neutron star's surface in a slow, "spot-less" accretion. This seems to be the case for the majority of LMXRBs for which no regular pulsations have been observed (see e.g. White et al. 1995; Psaltis 2004, for a review). In a few cases, though, regular X-ray pulsations have been detected with spin periods ranging from about $120 \mathrm{~s}$ for GX $1+4$ to less than $10 \mathrm{~ms}$, e.g. for SAX J1808.4-3658. The discovery of the latter accretionpowered millisecond pulsar (APMSP) has been very important in the LMXRB evolution scenario: the existence of such systems supports the theory that LMXRBs are indeed the progenitors of millisecond radio pulsars with a low magnetic field.
Currently seven APMSPs are known. A recent review of these systems can be found in Wijnands (2005) ${ }^{1}$. Among them IGR J00291+5934, with its $1.67 \mathrm{~ms}$ spin period, is the fastest known APMSP. Evidence for the existence of rapidly (ms) spinning neutron stars in a wider sample of LMXRBs (at least in an additional 11 systems) has been provided by the detection of nearly coherent oscillations ("burst oscillations") during type-I X-ray bursts (Chakrabarty 2004). Nevertheless, only for a few LMXRBs can we actually see the spin due to regular X-ray pulsations. The reason for this discrepancy is still debated, and it is not clear why the physics at the origin of the X-ray pulsations reveals itself only through the presence of pulsations and not in the spectral and timing properties of the sources that remain similar between APMSPs and nonpulsating LMXRBs (Wijnands 2005).

1 With the exception of the very recently discovered HETE J1900.1-2455 (Morgan et al. 2005). 
In this paper we report on observations of the fastest discovered APMSP, IGR J00291+5934, performed 12 days after the discovery, with the Chandra X-ray Observatory (Weisskopf et al. 2002). For a better overview of the spectral behaviour of the source prior to our observation, we also analysed the available Rossi X-ray Timing Explorer (RXTE; Jahoda \& PCA Team 1996) observations, part of which were simultaneous with our 18 ks Chandra observation.

The paper is organised as follows: in Sect. 2 we give an overview of current knowledge of IGR J00291+5934 from its discovery. In Sect. 3 we present our observations and data reduction methods. In Sect. 4 we present our results that are then discussed in the last section.

\section{IGR J00291+5934}

IGR J00291+5934 was discovered by the INTErnational Gamma-Ray Astrophysics Laboratory (INTEGRAL; Winkler et al. 2003) on December 2nd 2004, during routine monitoring of the Galactic plane (Eckert et al. 2004). Follow-up observations with $R X T E$ revealed the presence of coherent pulsations at $\sim 598.89 \mathrm{~Hz}$ (Markwardt et al. 2004) with an energy-dependent fractional amplitude. Further analysis and observations with $R X T E$ showed that the neutron star is in an $8844 \mathrm{~s}(2.45 \mathrm{~h})$ orbit. The upper limit of $0.16 M_{\odot}$ on the mass of the companion star in IGR J00291+5934 implies that the companion is most probably a hot brown dwarf (Galloway et al. 2005).

The outburst X-ray spectrum could be fitted with an absorbed power-law with $\Gamma=1.8$ and a fixed column density of $N_{\mathrm{H}}=2 \times 10^{21} \mathrm{~cm}^{-2}$ for INTEGRAL (Shaw et al. 2005) and $\Gamma=1.7$ and a measured column density $N_{\mathrm{H}}=1.7 \times 10^{21} \mathrm{~cm}^{-2}$ for RXTE (Galloway et al. 2005). More physical models (thermal Comptonisation) led to poorly constrained electron temperatures with a cut-off between $80-120 \mathrm{keV}$ (Shaw et al. 2005). Jonker et al. (2005) observed IGR J00291+5934 with Chandra one month after the discovery of the source, very likely at its quiescent flux level, and obtained a $0.5-10 \mathrm{keV}$ flux of $\sim 10^{-13} \mathrm{erg} \mathrm{cm}^{-2} \mathrm{~s}^{-1}$ and a neutron star effective temperature of $\sim 0.3 \mathrm{keV}$.

Inspection of the RXTE/ASM archive showed that the source was likely to be also active in the past on two occasions, leading to a tentative recurrence time of about 3 years (Remillard 2004). Meanwhile, observations with ground based telescopes allowed the counterparts at optical and radio wavelengths to be discovered (Pooley 2004; Fender et al. 2004; Fox \& Kulkarni 2004). The most accurate optical position of the source is RA $=00^{\mathrm{h}} 29^{\mathrm{m}} 03.06^{\mathrm{s}}$ and Dec $=+59^{\circ} 34^{\prime} 19.0^{\prime \prime}\left(0.5^{\prime \prime}\right.$ uncertainty) (Fox \& Kulkarni 2004).

\section{Observations and data reduction}

An X-ray intensity history of the 2004 outburst of IGR J00291+5934 is presented in Fig. 1, where the INTEGRAL detection, followed by the RXTE follow-up, are shown. The time of our Chandra observation is indicated by the arrow on the right. As can be seen, we observed the source at a very faint flux level, after the source had decayed significantly towards quiescence.

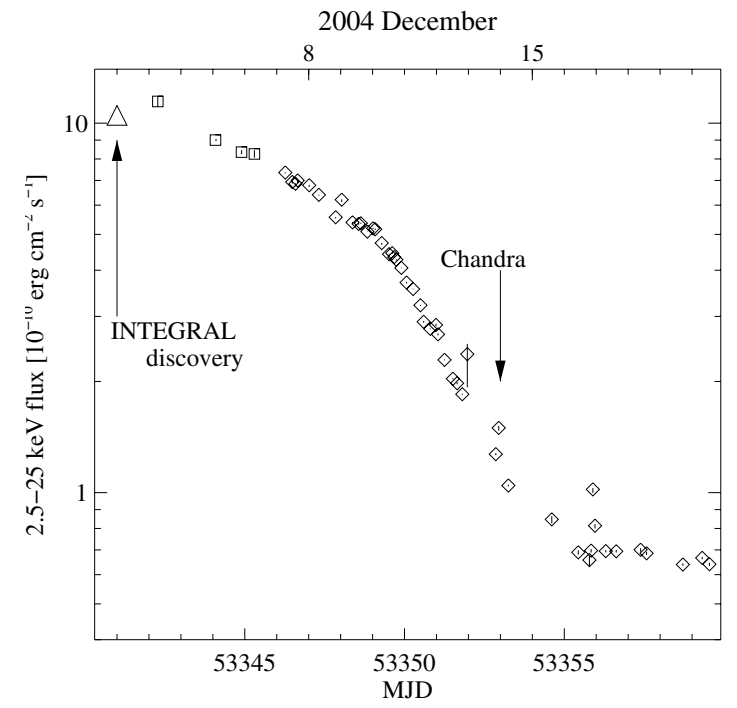

Fig. 1. X-ray intensity of IGR J00291+5934 throughout the 2004 outburst. The open triangle indicates the discovery with INTEGRAL. The 2.5-25 keV PCA flux evolution is shown (open squares). The time of our Chandra observation is indicated by the arrow on the right. After MJD 53355 the source flux is strongly influenced by residual flux and variability of (at least) the nearby V709 Cas.

\subsection{The Chandra data}

The $18 \mathrm{ks}$ Chandra observation of IGR J00291+5934 was performed on December 14th 2004 from 02:43:41 until 08:02:38 UT (MJD 53353). We reduced the data in a standard manner, using the CIAO version 3.2 software package and CALDB version 3.0.0. The obtained spectra were analysed with the ISIS analysis system, version 1.2.4 (Houck 2002).

We used the High Energy Transmission Grating Spectrometer, HETGS (Canizares et al. 2000). It has two sets of gratings, the High Energy Grating, HEG, and Medium Energy Grating, MEG, covering the energy ranges of $0.8-10 \mathrm{keV}$ and $0.4-5.0 \mathrm{keV}$, respectively. The focal plane imager used is the Advanced CCD Imaging Spectrometer (ACIS-S), an array of six CCD detectors normally used as readout for the photons dispersed by the gratings. The CCDs were operated in a sub-array mode where only half the CCD was read out. This did not affect the dispersed spectra, but served to reduce the frame integration time from the usual $3.2 \mathrm{~s}$ to $1.7 \mathrm{~s}$, and thus reduced the presence of pileup (see below).

Given the low signal-to-noise obtained, we extracted the zeroth-order (undispersed) spectrum and the first order dispersed spectra ( $m= \pm 1$ for HEG and MEG) for a total of five spectra. Higher order spectra were not considered. The zerothorder image of the source was well resolved, but is also mildly piled up (pile-up fraction of 18\%). In all spectral fits wherein we fit the zeroth-order data, we accounted for these effects by using the ISIS implementation of the pileup model of Davis (2001).

Within the field of view of ACIS-S we detect another source, $17^{\prime}$ away from the zeroth-order image of IGR J00291+5934 . The source is V709 Cas, a cataclysmic variable. The zeroth-order image of V709 Cas lies on top of the HEG $m=-1$ dispersed spectrum of IGR J00291+5934. 
The position of $\mathrm{V} 709$ Cas corresponds to the $1-1.3 \mathrm{keV}$ region of the HEG $m=-1$ spectrum, ignored in the spectral analysis. Outside of $1-1.3 \mathrm{keV}$, the removal of events associated with V709 Cas was extremely efficient and no contamination was occurring.

Inspection of the 5 spectra showed that the separate grating arms agree with each other. This allowed us to merge the two HEG $(m= \pm 1)$ and MEG $(m= \pm 1)$ spectra into two combined spectra in order to increase the signal-to-noise ratio. This led to the three final spectra that we used in the analysis (combined first order HEG, combined first order MEG, and zeroth-order). For the fit, we binned the data to obtain a minimum of 16 counts per bin for both HEG and MEG, as well as a minimum number of 16 channels per bin for HEG and 8 for MEG.

The $1.67 \mathrm{msec}$ pulsations cannot be detected in our data, due to the $1.7 \mathrm{~s}$ frame integration time in our observations. We used both "unbinned techniques" for searching for variability on all time scales $>1.7 \mathrm{~s}$ (e.g., the Bayesian Blocks method of Scargle 2005, in prep. ${ }^{2}$ ), as well as "binned" period folding techniques to search for variability on time scales comparable to the orbital period. No statistically significant variability was found.

\subsection{The RXTE data}

One day after the discovery of IGR J00291+5934, a regular monitoring of the source was performed with $R X T E$ via Target of Opportunity (ToO) observations starting from December 3rd until December 16th. The RXTE observation on December 14th was performed simultaneously to our Chandra pointing (from 03:08:48 to 08:53:52 UT). The RXTE data were extracted with the HEASOFT software, version 5.3.1 using our standard procedures (Wilms et al. 1999).

The simultaneous Chandra/RXTE fit was performed in the ISIS package already mentioned. We rebinned the HETGS spectra further to obtain a minimum number of channels per bin equal to 64 for HEG and 32 for MEG. In this way the number of PCA and HETGS data bins is comparable, and during the fit similar weight was given to all the available spectra (HEG, MEG, PCA). We fitted the PCA spectra in the $3-13 \mathrm{keV}$ spectral range with a $0.5 \%$ systematics and grouped the data to have a minimum signal-to-noise of 5. Interpretation of the PCA December 14 spectrum of IGR J00291+5934 is delicate due to the presence of (at least) the nearby cataclysmic variable V709 Cas (17' away, see Sect. 3.1) that was clearly detected in the Chandra field of view.

To take the contamination from nearby sources into account, we subtracted the last RXTE pointing available from all the previous observations. The integrated $2-8 \mathrm{keV}$ flux in this last PCA observation is consistent with the Chandra determined flux of V709 Cas alone $\left(2.4 \times 10^{-11} \mathrm{erg} \mathrm{cm}^{-2} \mathrm{~s}^{-1}\right)$, independently confirming the nondetection of IGR J00291+5934 in this observation.

\footnotetext{
${ }^{2}$ See the implementation of this algorithm in ISIS/s-lang at http://space.mit.edu/CXC/analysis/SITAR/
}

\section{Results}

We extracted the X-ray position of IGR J00291+5934 from the zeroth-order image, obtaining $\mathrm{RA}=00^{\mathrm{h}} 29^{\mathrm{m}} 03.08^{\mathrm{s}}$ and Dec $=+59^{\circ} 34^{\prime} 19.2^{\prime \prime}$ equinox J2000 (90\% confidence error of $\left.0.6^{\prime \prime 3}\right)$. This position, compatible with the optical and radio ones, was immediately announced to the community by Nowak et al. (2004).

The first order HEG and MEG spectra of IGR J00291+5934 are shown in Fig. 2. The best fit obtained, also shown in Fig. 2, is an absorbed power-law with column density ${ }^{4} N_{\mathrm{H}}=(4.3 \pm 0.4) \times 10^{21} \mathrm{~cm}^{-2}$ and $\Gamma=2.06 \pm 0.07$ with a reduced $\chi_{v}^{2}=0.79$ for 431 d.o.f. The absorbed flux is $2.5 \times 10^{-11} \mathrm{erg} \mathrm{cm}^{-2} \mathrm{~s}^{-1}$ in $0.5-8 \mathrm{keV}$ and $1.9 \times 10^{-11} \mathrm{erg} \mathrm{cm}^{-2} \mathrm{~s}^{-1}$ in $2-8 \mathrm{keV}(\sim 1 \mathrm{mCrab})$ with about $10 \%$ uncertainty.

Residuals in the continuum are found by taking the bestfit continuum model, and then using that as a "Bayesian prior" for the expected count rate in each bin of the unbinned spectrum. One can then apply the Bayesian Blocks algorithm of Scargle (2005, in prep.) to search for the most significant data residuals ${ }^{5}$. Using this procedure, we found the most significant residuals to be excess emission between approximately 6.4 and $6.8 \mathrm{keV}(1.94-1.82 \AA)$ and an emission deficit between 7.0 and $7.2 \mathrm{keV}(1.73-1.78 \AA)$, as shown in Fig. 3. Fitting just the HEG spectrum between 1.5 and $3 \AA$, where we have grouped the data uniformly by 7 bins, and using the statistic of Cash (1979), if we set the normalisation of the absorption line to zero and refit, the statistic increases by 10.8 . This is approximately the $99.3 \%$ significance threshold. Setting the normalisation of the emission line to zero and refitting only changes the Cash statistic value by 3 . Thus, barring systematic uncertainties of the detector, this absorption feature is approximately $3 \sigma$ significant. Hints for the presence of these featues are seen in the zerothorder spectrum as well, as we show below.

The simultaneous HETGS-PCA spectrum gave an overall harder spectrum with $\Gamma=1.8$. The harder continuum set by PCA gave a soft excess in the Chandra spectrum, which was then adjusted in the fit resulting in a smaller column density $\left(N_{\mathrm{H}}=3.1 \times 10^{21} \mathrm{~cm}^{-2}\right)$. Nevertheless the fit was not very good, reduced $\chi_{v}^{2}$ of 1.35 (163 d.o.f.), and indeed the low end of the MEG spectrum is not described well; the new $N_{\mathrm{H}}$ is lower than the value obtained fitting the Chandra data alone and predicts more photons in the soft end of the spectrum than what was actually detected by the MEG. Furthermore, the higher end of the PCA spectrum shows a slight excess above $8 \mathrm{keV}$, meaning that the 1.8 slope is not hard enough for the PCA data.

To adjust for these issues, we added a soft thermal part to the spectrum (DISKBB model, Mitsuda et al. 1984) obtaining a very good description of the data $\left(\chi_{v}^{2}=0.9,161\right.$ d.o.f. $)$ : the PCA data are better described by the new power-law $(\Gamma=1.7)$ and

\footnotetext{
3 See http://asc.harvard.edu/cal/ASPECT/celmon/ index.html

4 The improved model for the absorption of X-rays in the interstellar medium "tbvabs" Version 1.0 was used (Wilms et al. 2000).

5 This procedure is described in detail at: http: //space.mit.edu/CXC/analysis/SITAR/ bb_experiment.html
} 


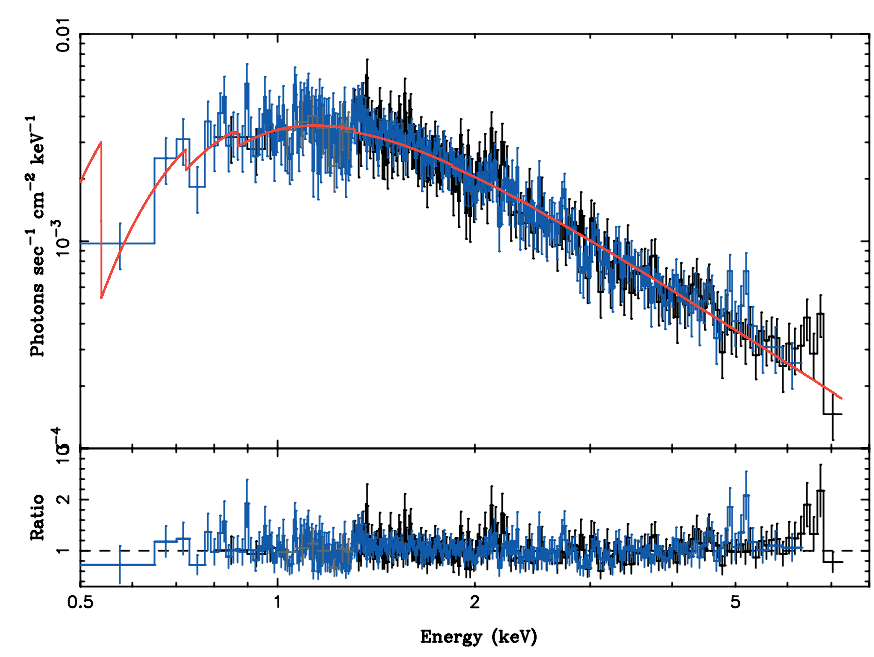

Fig. 2. Chandra HEG (order $m= \pm 1$ combined, black) and MEG (order $m= \pm 1$ combined, blue) spectra of IGR J00291 +5934 . The model shown is an absorbed power-law.

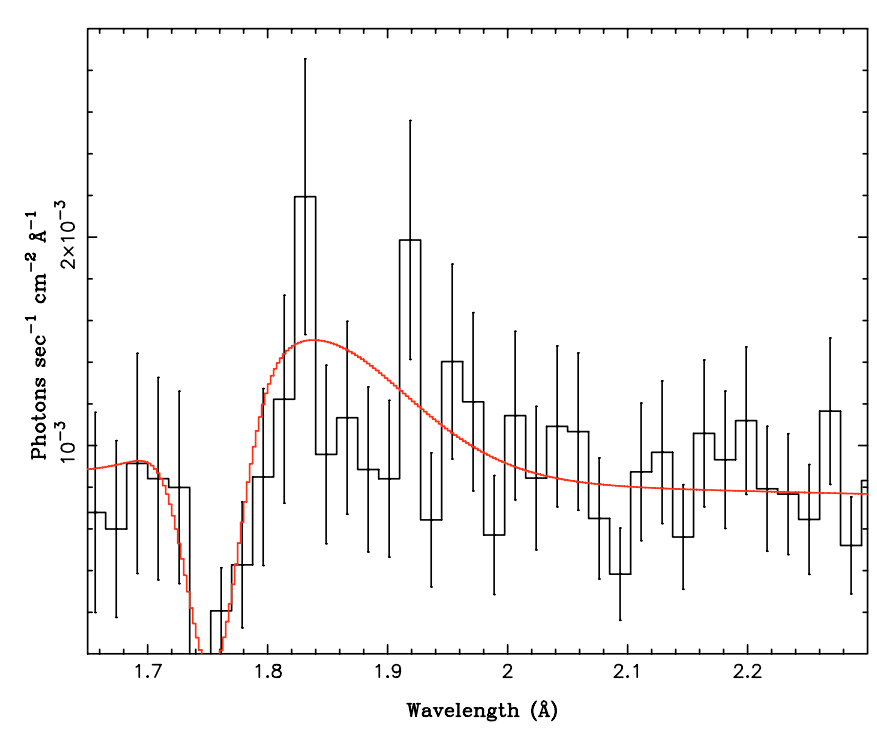

Fig. 3. HEG data uniformly rebinned by a factor of 7, where we fitted this region with a power law plus broad line plus absorption feature. The latter is the most significant feature in our HEG spectra (approximately $99.3 \%$ significant) and is also present in the zeroth-order spectrum.

the softer part of the spectrum is well fitted by both $N_{\mathrm{H}}=4.3 \times$ $10^{21} \mathrm{~cm}^{-2}$ and a thermal component of $k T=0.42 \mathrm{keV}(13 \%$ contribution to the absorbed $0.5-8 \mathrm{keV}$ flux) that compensates for the soft excess induced by the hard slope. Figure 4 shows the HEG, MEG, and PCA spectra of IGR J00291+5934 together with the best-fit model.

The discrete feature at $2.1 \mathrm{keV}$ is a known calibration origin and is due to a jump in the HETGS effective area. The excess at $\sim 5 \mathrm{keV}$ has no known instrumental origin and could be real; however, it is seen neither in the HEG nor in the zeroth-order spectrum and is less significant than the iron region features already discussed which are mirrored also in the zeroth-order spectrum.

We find that the normalisation of the PCA spectrum is about $13 \%$ higher than for Chandra. Between these two

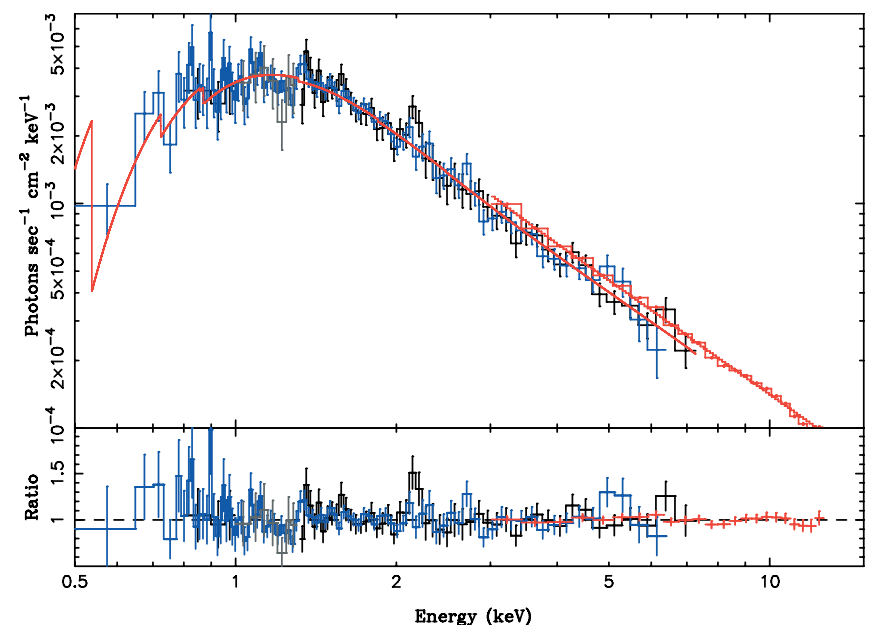

Fig. 4. HEG (black), MEG (blue), and PCA (red) spectra of IGR J00291+5934. The fit shown is a composition of an absorbed power-law and disc blackbody.

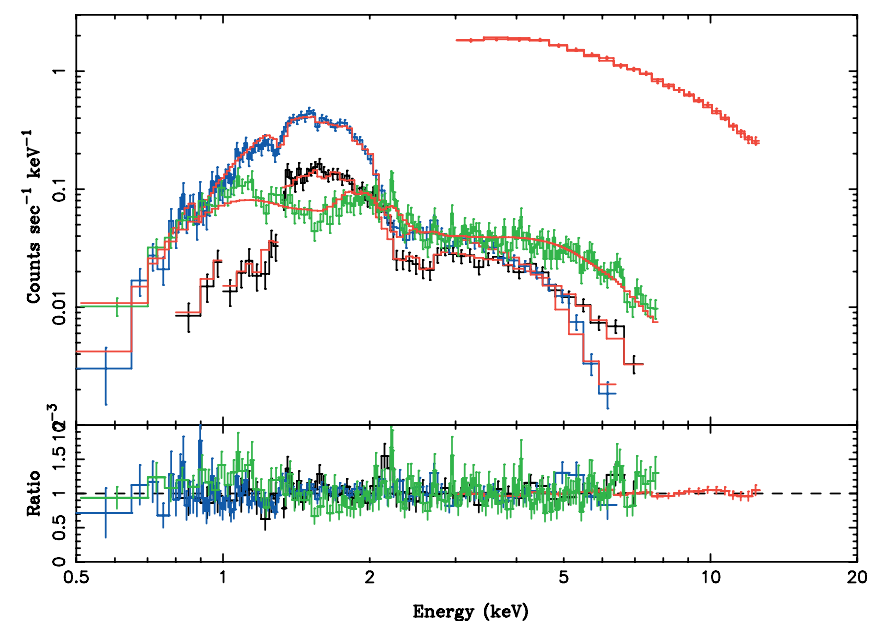

Fig. 5. HEG (black), MEG (blue), PCA (red), and HETGS zerothorder (green) count spectra of IGR J00291+5934. Note the emission/absorption line features already discussed (Fig. 3) in both the zeroth-order and HEG spectra. The break in the HEG spectrum (data points between $1-1.3 \mathrm{keV}$ ) is due to the fact that in this range we have used HEG $m=+1$ alone and not the averaged $m= \pm 1$ orders, due to the contamination by V709 Cas in the HEG $m=-1$ order.

missions, normalisation factors up to $20 \%$ have been found before (e.g., Juett et al. 2003, and references therein). We note here that if we use a non-cleaned PCA spectrum, i.e. not corrected for the contribution of the nearby sources, we obtain a normalisation factor of $50 \%$ with respect to Chandra. This high value is most likely due to the contamination from nearby sources and background in the PCA spectrum.

As a final step, we included the Chandra zeroth-order spectrum that is affected by pile-up such that the spectral shape from the source is distorted. Figure 5 shows the HEG, MEG, PCA and zeroth-order count spectra. The spectral distortion due to the pile-up in the zeroth-order spectrum is visible in the dip in the $1-2 \mathrm{keV}$ region in the green spectrum. The emission/absorption features detected by the HEG in the iron region (see Fig. 3) are mirrored in the zeroth-order as well. The 
Table 1. Best-fit parameters for the absorbed power-law model of the HETGS spectra of IGR J00291+5934 and for the absorbed power-law plus thermal component of the HETGS, PCA, and Chandra zerothorder spectra. $N_{\mathrm{H}}$ is the column density in units of $10^{21} \mathrm{~cm}^{-2} ; k T_{\text {in }}$ is the inner temperature of the thermal (DISKBB) component; d.o.f. = degrees of freedom (the decrease in d.o.f. in the combined HEG/MEG and PCA spectrum is due to the heavy rebinning we performed in the HETGS data, see text); in all the fits we fixed HEG, MEG and zerothorder normalisation factors to 1 . The indicated errors are at $1 \sigma$.

\begin{tabular}{lll}
\hline \hline & HEG / MEG & HEG / MEG / PCA \\
\hline$N_{\mathrm{H}}$ & $4.3 \pm 0.4$ & $4.3_{-0.5}^{+0.7}$ \\
$\Gamma$ & $2.06 \pm 0.07$ & $1.7 \pm 0.08$ \\
$k T_{\text {in }}$ & & $0.42_{-0.06}^{+0.07} \mathrm{keV}$ \\
PCA norm & $1.13^{2}$ \\
reduced $\chi_{v}^{2}$ & 0.79 (431 d.o.f.) & 0.9 (161 d.o.f.) \\
\hline \hline \multicolumn{3}{c}{ HEG / MEG } \\
& PCA / 0th order \\
\hline$N_{\mathrm{H}}$ & $3.9_{-0.4}^{+0.5}$ \\
$\Gamma$ & $1.7 \pm 0.04$ \\
$k T_{\text {in }}$ & $0.40 \pm 0.06 \mathrm{keV}$ \\
PCA norm & 1.13 \\
reduced $\chi_{v}^{2}$ & 1.15 (293 d.o.f.) \\
\hline
\end{tabular}

information that is lost due to pile-up can be partly recovered using Kernel models that "correct" the distortions (Davis 2001). Nevertheless, the result cannot be perfect and the inclusion of the zeroth-order spectrum can make the overall fit quality slightly worse. We obtained an absorbed power-law and thermal component, compatible with the previous case, with column density $N_{\mathrm{H}}=3.9 \times 10^{21} \mathrm{~cm}^{-2}, \Gamma=1.7, k T_{\text {in }}=0.4 \mathrm{keV}$, and a reduced $\chi_{v}^{2}=1.15$ for 293 d.o.f. Again we find a PCA normalisation of about $13 \%$ higher than for Chandra. Table 1 summarises the results of our spectral analysis.

To have an overview of the outburst behaviour of IGR J00291+5934 before our Chandra observation, we analysed all the available RXTE/PCA observations. The PCA spectra of IGR J00291+5934 can be fitted by a combination of a thermal component $(\sim 1 \mathrm{keV})$ and power-law $(\Gamma \sim 1.6)^{6}$. A hint of an iron line at $6.4 \mathrm{keV}$ (fixed) was detected. During the outburst, while the source was decaying, the power-law photon index showed no particular trend (unlike the normalisation that decreases significantly), while the thermal component became weaker and could be constrained no longer by PCA. Already two days before our Chandra observation, the RXTE observations were consistent with a single power-law with a marginal detection of the $6.4 \mathrm{keV}$ line. We believe that in our Chandra spectrum we still see a residual of the disappearing $6.4 \mathrm{keV}$ line, as well as a soft component $(0.4 \mathrm{keV})$ that cannot be constrained by PCA alone.

${ }^{6}$ This slope was adjusted to the softer 1.7 value when fitted with the Chandra simultaneous spectrum of December 14.

\section{Discussion}

We have studied the spectral evolution of the accretionpowered millisecond pulsar IGR J00291+5934 with Chandra and RXTE during the December 2004 outburst that led to its discovery by INTEGRAL.

\subsection{Long-term variability}

At its discovery, IGR J00291+5934 had a 5-100 keV flux of about $10^{-9} \mathrm{erg} \mathrm{cm}^{-2} \mathrm{~s}^{-1}$ (Shaw et al. 2005) that decayed to about $10^{-10} \mathrm{erg} \mathrm{cm}^{-2} \mathrm{~s}^{-1}$ (extrapolated from our HETGS/PCA best fit) about 12 days later. Assuming a distance of $5 \mathrm{kpc}$ (Galloway et al. 2005), we obtain that the X-ray luminosity changed from $L_{\mathrm{X}} \sim 10^{36} \mathrm{erg} \mathrm{s}^{-1}$ to $L_{\mathrm{X}} \sim 10^{35} \mathrm{erg} \mathrm{s}^{-1}$. This corresponds to a change from $\sim 0.01$ to $\sim 0.001 L_{\text {Edd }}$ that places IGR J00291+5934 at the lower end of the dim Atoll sources ( $\left.\sim 0.01-0.3 L_{\text {Edd }}\right)$, similar to other APMSPs. Such a peak luminosity is very low if we consider the bright neutron star $\mathrm{X}$-ray transients (XRTs) that display outbursts with peak luminosities of $L_{\mathrm{X}} \sim 10^{37}-10^{38} \mathrm{erg} \mathrm{s}^{-1}$. The history lightcurve of IGR J00291+5934 from the RXTE/ASM indicates that the flux measured at the time of the discovery is indeed the peak of the outburst; unfortunately, the distance to IGR J00291+5934 is not well constrained, and we cannot be sure of the absolute value. It may seem reasonable to expect the peak luminosity to be low because APMSPs are very compact systems and the disc outer radius $R$ is relatively small compared to wider orbit LMXRBs, which limits the total disc mass $\left(\propto R^{3}\right)$ that can build up in quiescence. When the outburst is triggered, the mass accretion onto the central object is $\propto R^{2}$ (Gierliński et al. 2002, and references therein). Nevertheless, things are not so straightforward, and there are cases where the scenario above does not hold, e.g. in the case of the ultra compact (11.4 min period) LMXRB 4U 1820-30 that can still reach X-ray luminosities higher than a few times $10^{37} \mathrm{erg} \mathrm{s}^{-1}$ (Ballantyne \& Strohmayer 2004).

APMSPs also differ from the bright XRTs in the quiescent luminosity: standard XRTs range between $L_{\mathrm{X}} \sim 10^{32}-10^{33} \mathrm{erg} \mathrm{s}^{-1}$ (Campana et al. 2002), while APMSPs seem to be dimmer than $\sim 10^{32} \mathrm{erg} \mathrm{s}^{-1}$ (Wijnands 2005). Our Chandra measurement of IGR J00291+5934 gives a $0.5-8 \mathrm{keV}$ flux of $\sim 10^{-11} \mathrm{erg} \mathrm{cm}^{-2} \mathrm{~s}^{-1}$ that, compared to the $0.5-10 \mathrm{keV}$ flux of $\sim 10^{-13} \mathrm{erg} \mathrm{cm}^{-2} \mathrm{~s}^{-1}$ by Jonker et al. (2005), clearly shows that we detected IGR J00291+5934 during its outburst decay, prior to its quiescent state.

\subsection{The spectral continuum}

The study of the other APMSPs has shown that during the outburst the spectra can be well fitted by a two component model, a soft thermal component and a harder one. In general the soft component is interpreted as blackbody emission from a heated hot spot on the neutron star, responsible for the X-ray pulsations, and it ranges within about $0.6-1 \mathrm{keV}$ (see e.g. Gierliński et al. 2002; Gierliński \& Poutanen 2005; Juett et al. 2003). The harder component is interpreted as thermal Comptonization by a plasma heated by the accretion shock as the material, 
collimated by the magnetic field, impacts onto the neutron star surface. The source of seed photons for Comptonisation seems to be either the hot spot itself (Gierliński \& Poutanen 2005) or the colder accretion disc (Titarchuk et al. 2002).

In our analysis, we found that the spectra of IGR J00291+5934 could be fitted by a combination of a thermal component and a power-law. Along the outburst, while the source was decaying, the power-law photon index showed no particular trend while the thermal component became weaker and could no longer be constrained by PCA. Our Chandra observation and the simultaneous weak Chandra/RXTE spectrum could be described well by the combination of a colder thermal component $\left(k T_{\text {in }}=0.4 \mathrm{keV}\right.$ instead of $\sim 1 \mathrm{keV}$ ) and a power-law. The presence of the power-law means that if there is a cut-off in the spectrum, then this occurs at higher energies than the HETGS/PCA range so we are not able to constrain it. A Comptonisation model in our data gave a poorly constrained Comptonising plasma temperature of about $50 \mathrm{keV}$. The presence of the thermal component means that not all the available soft photons go through the Comptonising medium; instead there are parts that are seen directly, similar to what was found in other APMSPs in outburst: Miller et al. (2003) and Gierliński \& Poutanen (2005) on XTE J1751-305, Gierliński et al. (2002) on SAX J1808.4-3658 and Juett et al. (2003) on XTE J0929-314.

We interpret the evolution we see in PCA data and the final simultaneous PCA/Chandra spectral properties in the following way: at the on-set of the outburst the accreting matter is channelled onto the neutron star surface and a hot spot is created. We have indications for the hot spot in the early PCA data with a thermal component around $1 \mathrm{keV}$ that is likely to be responsible for the pulsations detected by Galloway et al. (2005). The disc is colder $(<1 \mathrm{keV})$ and we do not detect it directly in the PCA spectra alone, but we see its effect in a hint of the $6.4 \mathrm{keV}$ line in the PCA data, most likely originating from irradiation of the cold accretion disc by the X-ray source ${ }^{7}$. Along the outburst, the hot spot becomes progressively weaker and, already 10 days after the on-set of the outburst, we are not able to constrain it with PCA anymore. This is consistent with the fact that Galloway et al. (2005) detect no pulsations in the last phases of the outburst. The disc is most likely becoming colder as it is illuminated by the fading X-ray source and is possibly receding after the outburst, similar to other LMXRBs in which, at a low accretion rate, the disc is more distant from the compact object and the overall spectrum is hard. At the time of our Chandra observation, the hot spot (along with its pulsations) was most likely off and we detected a cold accretion disc around $0.4 \mathrm{keV}$.

\footnotetext{
${ }^{7}$ A direct simultaneous measure of the hot spot and disc has been reported in the case of the APMSP XTE J1751-305 by Gierliński \& Poutanen (2005), where two soft components were detected in the broad-band XMM-Newton/RXTE spectrum: $\sim 0.6 \mathrm{keV}$, associated to the disc and $\sim 1 \mathrm{keV}$, the hot spot.
}

\subsection{Discrete features in the spectrum}

Despite the regular search for discrete features in APMSPs in the past, no line was found besides the $6.4 \mathrm{keV}$ one in SAX J1808.4-3658 (Gierliński et al. 2002). In our study, a hint of an iron $\mathrm{K} \alpha$ line (fixed at $6.4 \mathrm{keV}$ ) was detected in the PCA observations, while a marginally significant excess in the $6.4-6.8 \mathrm{keV}$ region and a $3 \sigma$ significance deficit around $7.1 \mathrm{keV}$ were detected in both the HEG and zeroth-order Chandra spectra (Figs. 3 and 5). Emission lines at $6.4 \mathrm{keV}$ were observed from many X-ray pulsars and are believed to be produced by fluorescence of weakly ionised iron (less than Fe XV). A likely candidate for this cold matter is the accretion disc that can also be responsible for the iron K-edge $\sim 7.1 \mathrm{keV}$ feature we detect in the spectrum. The emission line that we detect around $6.8 \mathrm{keV}$ (if real) would require the presence of a highly ionised, i.e. hot, corona in IGR J00291+5934, consistent with the high plasma temperature $(>10 \mathrm{keV})$ inferred from the spectral study performed here, as well as in Shaw et al. (2005). This scenario would require a different medium for each feature of Fig. 3 (cold disc for the $7.1 \mathrm{keV}$ feature and hot corona for the $6.8 \mathrm{keV}$ one).

Another possible, although speculative, interpretation of the discrete features in Fig. 3 could be the following: the emission feature we detected around $6.8 \mathrm{keV}$ could be the redshifted part of the $6.97 \mathrm{keV}$ line (expected from Fe XXVI), while the absorption feature around $7.1 \mathrm{keV}$ could be the blueshifted $6.97 \mathrm{keV}$ line. In this scenario we would basically find that the $6.4 \mathrm{keV}$ line is produced by the cold disc while the 6.8 and $7.1 \mathrm{keV}$ lines are a P-Cygni profile from an expanding hot corona with outflow velocities of about $6000 \mathrm{~km} \mathrm{~s}^{-1}$. Such (and higher) outflow velocities are possible and have already been observed in cataclysmic variables (e.g., Woods et al. 1992) and in the X-ray binary SS433 (Migliari et al. 2005). The P-Cygni profile scenario is consistent with all the available data we currently have on IGR J00291+5934. However, the relatively low statistical significance of the discrete features does not allow us to firmly establish this interpretation and underlines the importance of obtaining quick follow-ups of these transient events.

Acknowledgements. We would like to thank the Chandra team for their help during the trigger of the ToO, and Jean Swank and Evan Smith for performing the RXTE observation simultaneous to our Chandra one. A.P. thanks J. Poutanen for sharing his overview knowledge of the physics of APMSPs and S. Shaw for careful reading of the manuscript. A.P. and P.U. acknowledge the Italian Space Agency financial and programmatic support via contracts ASI-I/R/046/04. MN was supported by NASA grant SV3-73016. This work was partly supported by NASA Grant GO4-5049X.

\section{References}

Ballantyne, D. R., \& Strohmayer, T. E. 2004, ApJ, 602, L105

Campana, S., Stella, L., Gastaldello, F., et al. 2002, ApJ, 575, L15

Canizares, C. R., Huenemoerder, D. P., Davis, D. S., et al. 2000, ApJ, 539, L41

Cash, W. 1979, ApJ, 228, 939

Chakrabarty, D. 2004, Binary Radio Pulsar ASP Conf. Ser. [arXiv: astroph-0408004]

Davis, J. E. 2001, ApJ, 562, 575 
Eckert, D., Walter, R., Kretschmar, P., et al. 2004, The Astronomer's Telegram, 352, 1

Fender, R., De Bruyn, G., Pooley, G., \& Stappers, B. 2004, The Astronomer's Telegram, 361, 1

Fox, D. B., \& Kulkarni, S. R. 2004, The Astronomer's Telegram, 354, 1

Galloway, D. K., Markwardt, C. B., Morgan, E. H., Chakrabarty, D., \& Strohmayer, T. E. 2005, ApJ, 622, L45

Gierliński, M., Done, C., \& Barret, D. 2002, MNRAS, 331, 141

Gierliński, M., \& Poutanen, J. 2005 [arXiv: astro-ph/0411716]

Houck, J. C. 2002, in High Resolution X-ray Spectroscopy with XMM-Newton and Chandra

Jahoda, K., \& PCA Team. 1996, Bull. Amer. Astron. Soc., 28, 1285

Jonker, P. G., Campana, S., Steeghs, D., et al. 2005 [arXiv:astro-ph/0505120]

Juett, A. M., Galloway, D. K., \& Chakrabarty, D. 2003, ApJ, 587, 754

Markwardt, C. B., Swank, J. H., \& Strohmayer, T. E. 2004, The Astronomer's Telegram, 353, 1

Migliari, S., Fender, R. P., Blundell, K. M., Méndez, M., \& van der Klis, M. 2005, MNRAS, 358, 860

Miller, J. M., Wijnands, R., Méndez, M., et al. 2003, ApJ, 583, L99

Mitsuda, K., Inoue, H., Koyama, K., et al. 1984, PASJ, 36, 741

Morgan, E., Kaaret, P., \& Vanderspek, R. 2005, The Astronomer's Telegram, 523, 1
Nowak, M. A., Paizis, A., Wilms, J., et al. 2004, The Astronomer's Telegram, 369, 1

Pooley, G. 2004, The Astronomer's Telegram, 355, 1

Psaltis, D. 2004, Compact Stellar X-ray Sources, ed. W. H. G. Lewin \& M. van der Klis, to appear [arXiv:astro-ph/0410536]

Remillard, R. 2004, The Astronomer's Telegram, 357, 1

Scargle, J. D. 2005, in preparation

Shaw, S. E., Mowlavi, N., Rodriguez, J., et al. 2005, A\&A, 432, L13

Titarchuk, L., Cui, W., \& Wood, K. 2002, ApJ, 576, L49

Weisskopf, M. C., Brinkman, B., Canizares, C., et al. 2002, PASP, 114, 1

White, N. E., Nagase, F., \& Parmar, A. N. 1995, From X-Ray Binaries, ed. W. E. G. Lewin, J. van Paradijs \& van den Heuvel E. P. J., Cambridge

Wijnands, R. 2005, Nova Science Publishers (NY) volume Pulsars New Research [arXiv: astro-ph/0501264]

Wilms, J., Allen, A., \& McCray, R. 2000, ApJ, 542, 914

Wilms, J., Nowak, M. A., Dove, J. B., Fender, R. P., \& di Matteo, T. 1999, ApJ, 522, 460

Winkler, C., Courvoisier, T. J.-L., Di Cocco, G., et al. 2003, A\&A, 411, L1

Woods, J. A., Verbunt, F., Collier Cameron, A., Drew, J. E., \& Piters, A. 1992, MNRAS, 255, 237 